\title{
Methane hydrates bearing synthetic sediments-Experimental and numerical approaches of the dissociation
}

TONNET, N. ; HERRI, J .M. ${ }^{(*)}$

(1) Département GENERIC (Géochimie, ENvironnement, Ecoulement, Réacteurs Industriels et Cristallisation), Centre SPIN. LPMG UMR CNRS 5148. Ecole Nationale Supérieure des Mines de Saint-Etienne 158, Cours Fauriel. 42023 Saint-Etienne Cedex 2.

(*) herri@emse.fr

Keywords:

Methane hydrate; Dissociation; Sediment core; Heat and mass transfer

\begin{abstract}
The production of methane gas from methane hydrate bearing sediments may reach an industrial scale in the next decades owing to the huge energy reserve it represents.

However the dissociation of methane hydrate in a porous medium is still poorly understood and controlled: the melting of methane hydrate involves fluids flows and heat transfer through a porous medium whose properties evolve as the hydrate phase disappears, and is replaced (or not) by an ice phase. Mass and heat transfers can be coupled in a complex way, firstly because of the permeability changes, and secondly due to material conduction changes. In our work, mass and heat transfers have been studied both experimentally and numerically.

A 2D numerical model is proposed where heat and mass transfers govern the dissociation of methane hydrate. This model has been used to design an experimental device. Experiments have been obtained and finally the model has been validated.

The experimental set-up consists of five cylindrical sand packs having the same diameter but different lengths. Each experiment starts by crystallizing a hydrate phase in a porous medium. Then the hydrate is dissociated by controlling the pressure at one boundary. The kinetic of dissociation is monitored by collecting gases in ballast. Simulations and experiments demonstrate that the dissociation limiting step switches from thermal transfer to mass transfer depending on the initial permeability and conductivity of the porous medium.
\end{abstract}

\section{Introduction}

The methane hydrates bearing sediments are the focus of a special attention as a potential energy resource in the next decades. These solids, composed of water and gas, are indeed present in the marine or continental sediments, and they represent bigger fossil fuel reserves than the known reserves of oil, gas and coal.

The dissociation of these compounds to produce gas induces significant fluid movements, thermal exchange, and mechanical impacts on the reservoir. To evaluate the potential for gas recovery in such fields, one needs to model all these effects. The present paper concentrates on the thermal and mass transfers during the dissociation of methane gas hydrate. The main challenge here is to account for a mobile boundary between the solid which melts and the remainder of the sediment. Mechanical effects induced by phase changes on sediment integrity or compaction of the reservoirs (Rutqvist et al., 2009) are not investigated.

Many production schemes have been proposed for hydrate gas reservoirs in which different processes of dissociation are computed. 
The thermal process, i.e. production assisted by hot water injection, was suggested in several studies (Durgut and Parlaktuna, 1996). The use of chemicals to dissociate hydrates by changing their condition of stability was also tested (Sung et al., 2002). However, the method of depressurization seems the most reliable and probably the most profitable (Yousif et al., 1991).

Few studies addressed the dissociation of hydrate in terms of boundary displacement. In the first ones (Ahmadi et al., 2004; Ji et al., 2001), the classical Stefan's problem for melting is used to describe hydrate decomposition, but pressure is set to obey a diffusion equation. Taking into account the gas and water flow into the porous medium allows to better describe the evolution of the system and to record the evolution of the dissociation zone (Yousif et al., 1991). Moreover, as decomposition takes place in a porous medium and as solid hydrate dissociates, permeability changes must be calculated and permeability updated during this phenomenon (Larsen et al., 2005).

Implicit pressure and explicit saturation (IMPES) method was developed for modeling the two phase flow model in a porous matrix (Chen et al., 2004). It is used to accelerate convergence of resolution, but it has been shown to fail on few problems. A fully implicit resolution (Chounet et al., 1999) is used in this paper to ensure convergence of results. Some schemes, such as the Koren upwind scheme (Holstad, 2001) are useful to satisfy conservation properties for the advection terms because of accuracy and stability. The resolution is further refined by allowing the system of equations to be reformulated with more appropriate master variables once a new phase appears or is exhausted (Bastian, 1999; Class, 2001; Class et al., 2002).

The influence of different parameters (porosity, permeability, initial pressure,...) on the dissociation of hydrate accumulations in oceanic sediments have been evaluated numerically (Moridis and Sloan, 2007). X-ray computed tomography was used to visualize local density changes during hydrate formation and dissociation in a core-scale sand sample (Kneafsey et al., 2007).

This paper builds on two previous works from the same group (Bonnefoy, 2005; Nguyen Hong, 2005). It addresses the simultaneous mass and heat transfers during the dissociation of a sediment core partially saturated in methane hydrate. A 2D multi-phase multicomponents model describes the flow of water and gas and the heat transfer in the sediment and helps understanding the mechanisms of dissociation. The model is then used to design an experimental set-up where different sediment types have been tested. All sediments used are loose sand packs, and consolidated sandstone cores have not been tested. More generally, the mechanical effects of hydrate formation-dissociation cycles are not evaluated in the present study. Experimental results document the influence of distinct parameters on the kinetics of dissociation. The paper is concluded with a comparison between numerical and experimental results.

\section{Model approach}

\section{Physical system and gas hydrate dissociation}

The description of the system, the different phases and components, and interactions, is the first step. Our system is composed of three phases (liquid, gas, hydrate) and three components (water, methane, hydrate). A local thermal equilibrium is assumed since flow velocities are small. Chemical equilibrium between dissolved and free gas is also assumed. 
Capillary effects in the porous medium are neglected since the average grain size is $250 \mu \mathrm{m}$ (Anderson et al., 2001).

In this study, we consider a sediment core partly saturated with methane hydrate to which we apply a depressurization to dissociate the methane hydrates.

At the beginning and during all the dissociation, water and free gas are present and allowed to flow in the porous medium in response to pressure and temperature gradients. Fig. 1 gives a schematic representation of the system and explains the dissociation phenomenon where hydrate releases water and free gas in the sediment according to

$\left[\mathrm{CH}_{4}\left(\mathrm{H}_{2} \mathrm{O}\right)_{6}\right]_{\text {hydrate }} \rightarrow \mathrm{CH}_{4}+6 \mathrm{H}_{2} \mathrm{O}$
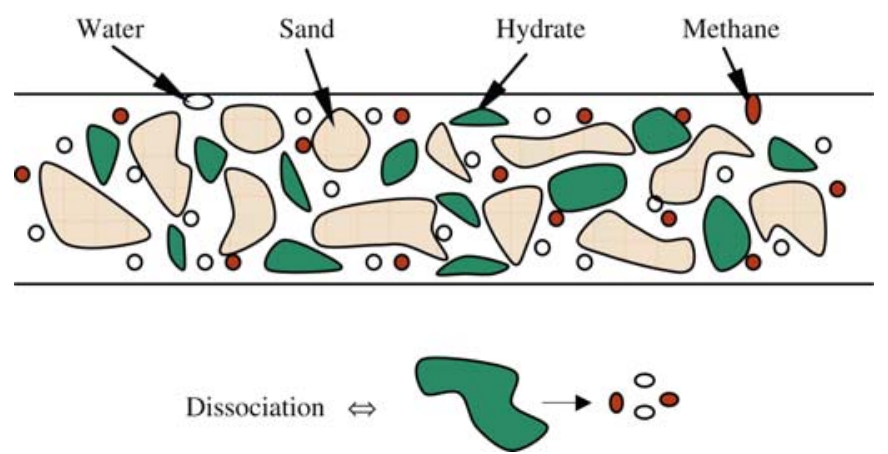

Fig 1: Physical system and description of the dissociation phenomenon

For the water phase, we assume the concentration of the dissolved component (methane) to be low but we do not neglect it. Dissolution and degassing are taken into account as mass transfers and the adsorption on solid surfaces is neglected (Fig. 2).

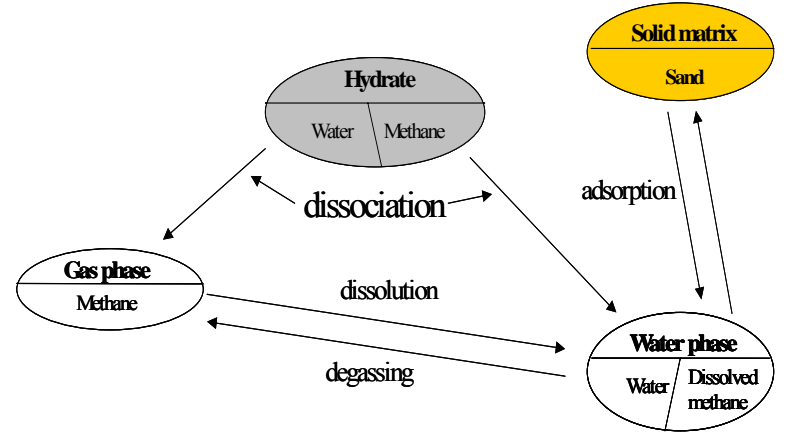

Fig 2: Interactions between different phases

\section{System parameters}

To find the equations of the problem, and to solve it by numerical resolution, it is convenient to distinguish two kinds of variables: the primary ones, which fully describe the evolution of the system and the secondary ones which are dependent on the primary. The number of these variables depends on the number of phases.

In the hydrate zone, four primary variables are used: temperature, pressure and saturations, i.e. the volume fractions of hydrate and water. Secondary variables are constrained by various models:

* The volumetric saturation balance is used to calculate the gas saturation:

$\sum_{j=l, g, h} S_{j}=1$ 
* The relative permeabilities for the two phase flow are expressed as functions of the reduced saturations $S^{\prime}$ defined as follows:

$S_{l, g}^{\prime}=\frac{S_{l, g}}{1-S_{h}}$

* The Brooks and Corey model is used:

$k_{r l}=S_{l}^{14}$

$k_{r g}=S_{g}^{\prime 2} \cdot\left(1-\left(1-S_{g}^{\prime}\right)^{2}\right)$

* To compute the evolution of the absolute permeability $K$ during the dissociation, the Civan law is used. $K$ and the absolute permeability of sediment $K_{\mathrm{o}}$ without hydrate are related by

$$
\frac{K}{K_{0}}=\frac{\phi_{e}}{\phi_{0}}\left(\frac{\phi_{e}\left(1-\phi_{0}\right)}{\phi_{0}\left(1-\phi_{e}\right)}\right)^{2 \beta}
$$

where $\varphi_{\mathrm{o}}$ and $\varphi_{e}$ represent the porosity and the effective porosity of the sediment $\left(\varphi_{e}=\varphi_{0}\left(S_{g}+S_{l}\right)\right)$, and $\beta$ is a parameter which allows to tune the impact of the hydrate saturation on the local permeability $K$ (Fig. 3). It is the only user-defined parameter in this model.

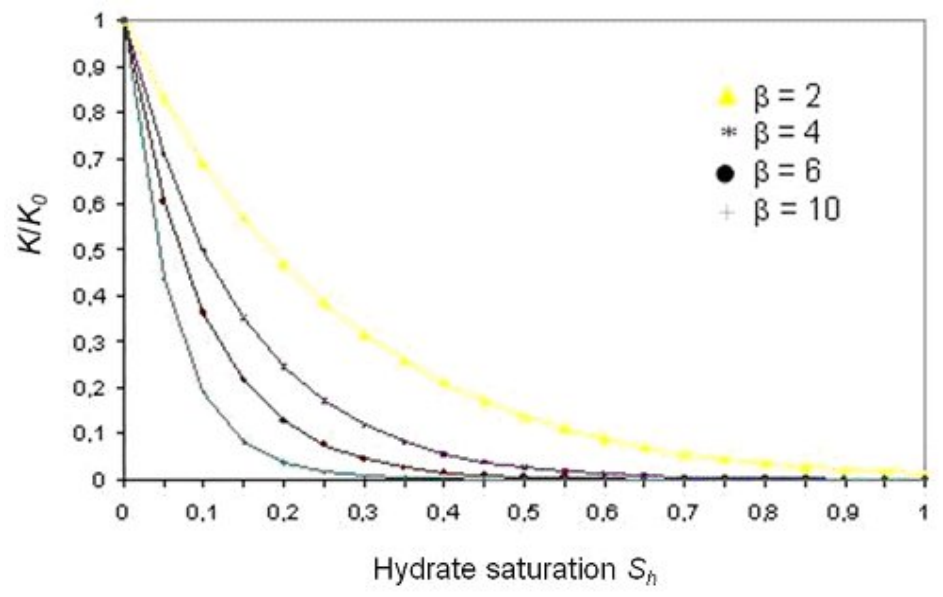

Fig 3:Evolution of the permeability $K$ with the $\beta$-parameter.

* The Heat conductivity $\lambda^{*}$ is estimated from the geometric model of (Henninges et al., 2002 )

$\lambda^{*}=\left(\Pi \lambda_{j}^{v_{j}}\right)^{1 / \sum v_{j}}$

where $\lambda \mathrm{j}$ is the heat conductivity and $\mathrm{vj}$ the volume fraction of the phase $\mathrm{j}$ (i.e. saturation).

* A Soave-Redlich-Kwong equation of state (Bonnefoy, 2005) is used to calculate the fluid density and the compressibility factor. The diffusion of methane dissolved in the water phase is taken into account in the equations of conservation; the diffusivity $\mathrm{D}$ is calculated from the Wilke and Chang's correlation (Herri, 1996). The percentage of methane in the water phase does not exceed $1 \%$ but can however have an influence on the system, particularly on the dissociation kinetics of methane hydrate. 


\section{Dissociation kinetics}

The kinetic description of the dissociation rate is a key point in the model. In this simulation, the rate of hydrate decomposition was computed according to the Kim-Bishnoï model (Kim et al., 1987) in which the driving force of the dissociation is the difference between the equilibrium and the actual gas fugacity. The net mass rate of hydrate dissociation writes:

$\gamma_{h}=k_{d} A_{S}\left(f_{e}-f\right)$

In this relation, the kinetic constant $k_{d}$ and the surface area $A_{S}$ are expressed as

$k_{d}=k_{d}^{0} \exp \left(-\frac{\Delta E}{R T}\right)$

where $\Delta E$ is the activation energy, $k_{d}^{0}$ the intrinsic kinetic constant, and the available surface for reaction $A_{s}$ is assumed to be the pore surface (i.e. the entire surface of the porous medium is covered with hydrate).

A Soave-Redlich-Kwong equation of state is also used to calculate the fugacity $f$ of gas; $\gamma_{l}$ and $\gamma_{\mathrm{g}}$ are derived from $\gamma_{h}$ using stoichiometric coefficients and molar masses. For the values of physical data in these expressions or more information about this model, see [17].

\section{Governing equations}

The velocity fields are calculated from the generalized Darcy's law and integrated into the mass balance equation (Eulerian approach) as

$\frac{\partial\left(\phi_{0} S_{\alpha} \rho_{\alpha}\right)}{\partial t}-\operatorname{div}\left(\rho_{\alpha} \frac{k_{r \alpha}}{\mu_{\alpha}} K\left(\operatorname{grad} p-\rho_{\alpha} g\right)\right)=0$

Individual mass balances for each component $k$ ( $k=$ water, methane, hydrate) writes

* Conservation of water:

$\phi_{0} \frac{\partial}{\partial t}\left[\left(\rho_{l} x_{l}^{e} S_{l}\right)\right]-\operatorname{div}\left(\frac{k_{r l}}{\mu_{l}} \rho_{l} x_{l}^{e} K\left(\operatorname{grad} p-\rho_{l} g\right)\right)-\operatorname{div}\left(D_{e} \rho_{l} \operatorname{grad} x_{l}^{m}\right)-\gamma_{l}=0$

* Conservation of methane gas:

$\phi_{0} \frac{\partial}{\partial t}\left[\left(\rho_{g} S_{g}\right)\right]-\operatorname{div}\left(\frac{k_{r g}}{\mu_{g}} \rho_{g} K\left(\operatorname{grad} p-\rho_{g} g\right)\right)+\phi_{0} \frac{\partial}{\partial t}\left[\left(\rho_{l} S_{l} x_{l}^{m}\right)\right]$

$-\operatorname{div}\left(\frac{k_{r l}}{\mu_{l}} \rho_{l} x_{l}^{m} K\left(\operatorname{grad} p-\rho_{l} g\right)\right)-\operatorname{div}\left(D_{m} \rho_{l} \operatorname{grad} x_{l}^{m}\right)-\gamma_{g}=0$

where $x_{l}^{m}$ is the mass fraction of methane dissolved in the liquid phase and : $x_{l}^{m}+x_{l}^{e}=1$ (Conservation of hydrates):

$\rho_{h} \phi_{0} \frac{\partial}{\partial t}\left[S_{h}\right]-\gamma_{h}=0$

where $\gamma_{l}, \gamma_{g}, \gamma_{h}$ represent, respectively, the mass net reaction rates of water, methane gas and hydrate due to chemical reaction (dissociation).

A thermal energy balance is written as 
$\frac{d E}{d t}=\frac{d Q}{d t}+\frac{d W_{V}}{d t}+\frac{d W_{D}}{d t}$

where the change of the internal energy $E$ equals the sum of the thermal energy flux $Q$ across the system boundaries, the work related to volume change $W_{V}$ and the dissipation work $W_{D}$. The hypothesis of local thermal equilibrium allows us to write a single balance of thermal energy for the entire domain, neglecting heat transfers between the fluid phases. Integrating the contribution of each phase a (fluid and solid) to the thermal energy balance in an elementary volume of the porous medium gives

$$
\begin{aligned}
& \phi_{0} \frac{\partial\left(\sum_{\alpha} \rho_{\alpha} e_{\alpha} S_{\alpha}\right)}{\partial t}+\left(1-\phi_{0}\right) \frac{\partial \rho_{s} c_{s} T}{\partial t}-\sum_{\alpha} \operatorname{div}\left\{\frac{k_{r \alpha}}{\mu_{\alpha}} \rho_{\alpha} h_{\alpha} K\left(\operatorname{grad} p_{\alpha}-\rho_{\alpha} g\right)\right\} \\
& -\sum_{k} \operatorname{div}\left(D_{k} \rho_{l} h_{k} M^{k} \operatorname{grad} x_{l}^{k}\right)-\operatorname{div}\left(\lambda^{*} \operatorname{grad} T\right)=0
\end{aligned}
$$

where $h$ is the specific enthalpy, $e$ the specific internal energy and $c_{S}$ the specific heat capacity of the sediment. The latent heat of methane hydrate dissociation $L_{h}$ appears in its specific enthalpy (the value is $L_{h}=4.246 \times 10^{5} \mathrm{~J} \mathrm{~kg}^{-1}$ at $T=273.15 \mathrm{~K}$ ).

\section{Numerical resolution}

Boundary and initial conditions

The objective of the calculation is to track mass and heat transfers for a given system. A cylindrical geometry is the most adapted one for this problem hence a sediment core is considered and defined with a length bigger than the diameter ( $\mathrm{L}>>d$, Fig. 4), and the dimensionless value $L / d$ as the main geometric parameter.

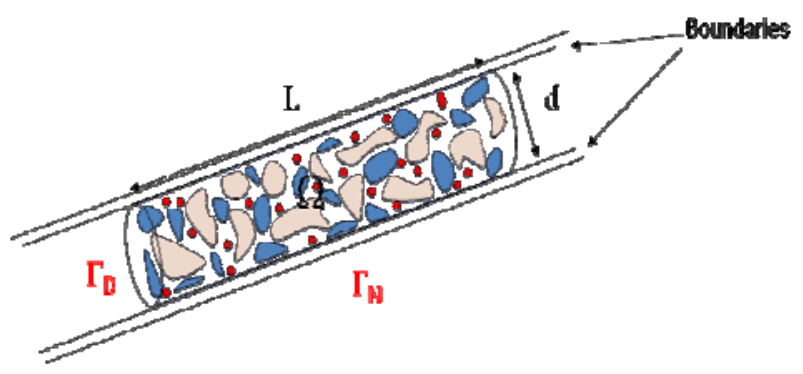

Fig 4: Description of boundaries.

Considering the domain $\Omega$ (the sediment zone), two distinct boundaries must be considered (Fig. 4). The boundary $\Gamma_{D}$ is a moving boundary where only the pressure is known (the pressure at the end of the pipe is controlled by the operator to dissociate the methane hydrate).

Concerning the boundary $\Gamma_{N}$, a mixed condition is retained: the temperature (or the heat flux) and the mass flux across it in the normal direction are known and set to zero.

Concerning the initial conditions, we assume that the sediment and the distribution of hydrates are homogeneous at the beginning of the dissociation. All the previously defined parameters can be changed but, two conditions are kept constant in the simulations:

* porosity $\varphi$ of the sediment is $37 \%$ and

* gas hydrate water equilibrium prevails in the sediment core before the dissociation, i.e. $P_{e}=3.55 \mathrm{MPa}$ at $T_{e}=276 \mathrm{~K}$. 


\section{Discretization and iteration method}

The mass conservation equations of water, methane gas, hydrate and energy constitute a system of four coupled partial differential equations which has a parabolic/hyperbolic character and a high degree of non-linearity.

As there are equations of conservation, spatial discretization is best achieved by integration on a control volume and the use of a volume finite scheme to ensure convergence and accuracy. A finite volume difference method (FVDM) is implemented. Moreover, an upwind scheme is applied for the advective flux coefficient (Holstad, 2001). For the time discretization, a fully implicit method (Euler) is used on account of the non-linear terms and the degree of coupling.

With regard to the numerical method, a Newton-Raphson approach is used. Following Saad (2000), we implemented the inexact Newton algorithm (Dawson et al., 1997) combined with the GMRES solver with restarting and an approximation of the Jacobian. This algorithm allows us to increase the time step and accelerate the convergence. For each time step, a verification of phases (in the entire domain) is needed. If somewhere in the domain a phase disappears, a change of a primary variable can be needed. Fig. 5 gives an overview of this algorithm.

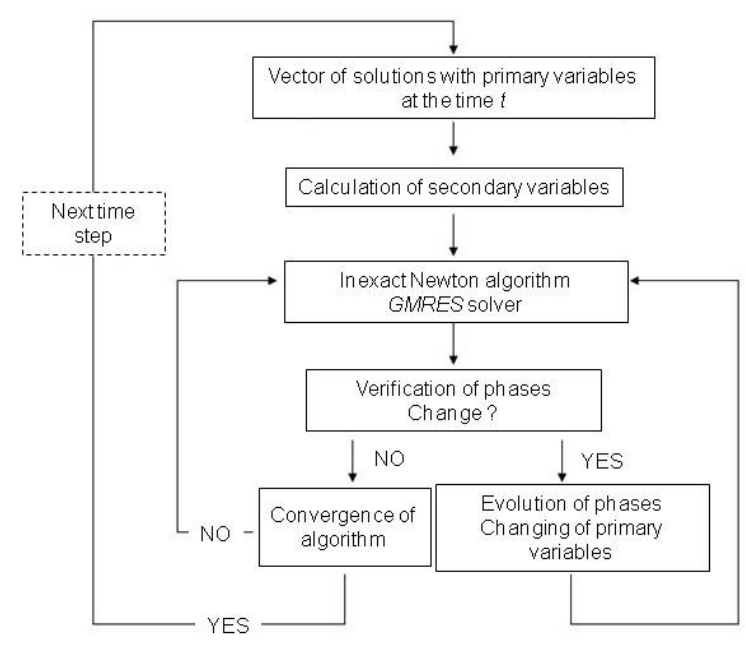

Fig 5: Algorithm of calculation.

With regard to the grid, an adaptive time step is used with a dichotomy method in order to minimize the number of iterations; moreover, an adaptive mesh size is also computed in order to have smaller meshes around the frontier between the hydrate and the non-hydrate zones. Indeed like this zone is highly disturbed (pressure or temperature gradients very important can be observed) a finer mesh is needed to limit error and to ensure convergence (Fig. 6). This mesh evolves with time (Fig. 7) and with the frontier movement.

Results and comments

In this section, some numerical results are given to illustrate the evolution of pressure, temperature and hydrate saturation in the sediment during the dissociation. For the next Fig. 8 and Fig. 9 we consider that depressurization is applied and dissociation begins at time $t=0$. Before each depressurization, the system is considered to be at equilibrium (i.e. the operator fixes initial pressure according to the chosen temperature and the equilibrium curve for methane hydrate). 


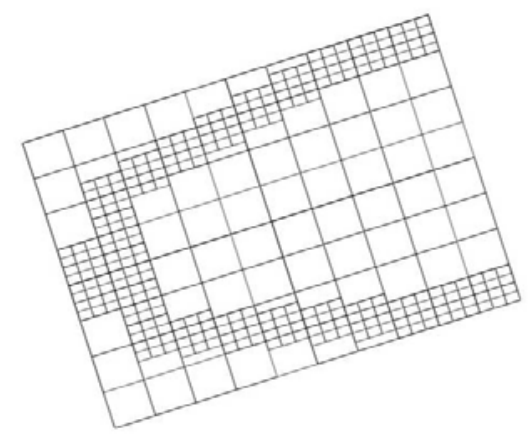

Fig 6: Adaptive mesh.

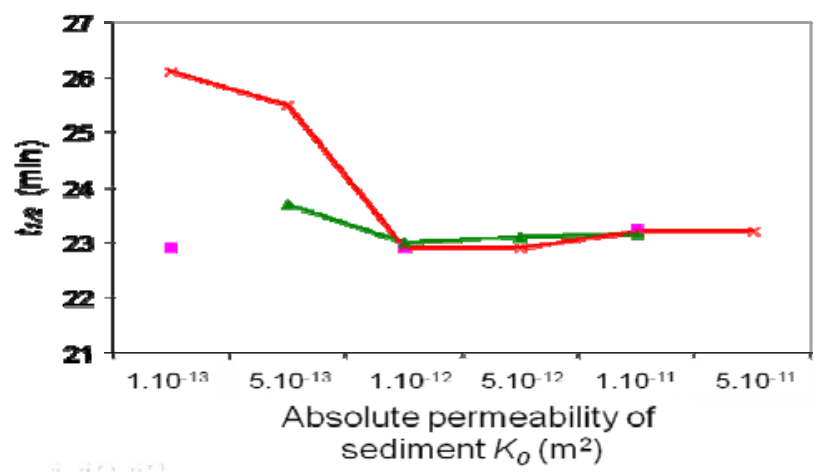

Fig 7: Evolution of time of half dissociation $t_{1 / 2}$ with absolute permeability $K_{o}(L / d=12,25,50$, $\left.\Delta P m=0.51 \mathrm{MPa}, \mathrm{S}_{\mathrm{h}}^{0}=0.5, \lambda=1.4 \mathrm{Wm}^{-1} \mathrm{~K}^{-1}\right)$.
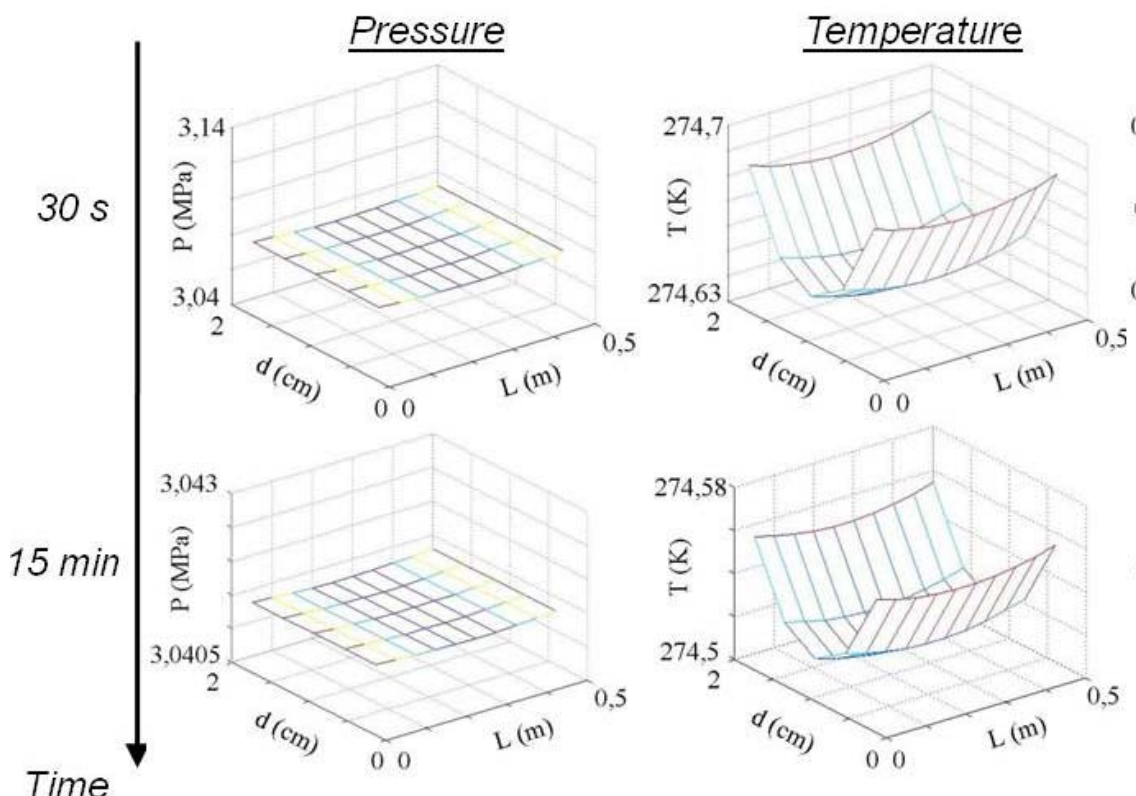

\section{Hydrate saturation}
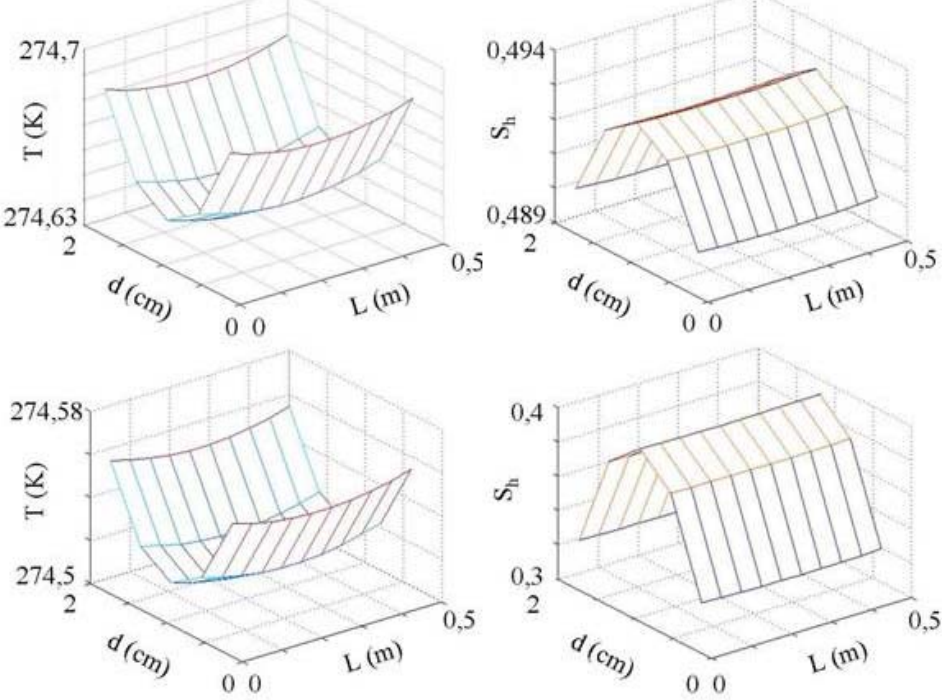

Fig 8: Evolution of pressure, temperature and hydrate saturation with time $\left(S_{h}{ }^{0}=0.5, S_{g}{ }^{0}=0.5, P_{d}=\right.$ $\left.3.04 \mathrm{MPa}, K_{0}=10^{-11} \mathrm{~m}^{2}, \lambda_{s}=1.4 \mathrm{~W} \cdot \mathrm{m}^{-1} \cdot \mathrm{K}^{-1}\right)$

We have selected two contrasting cases to illustrate different modes of dissociation of methane hydrate inside sediment cores. There is no free water in the system before the dissociation, temperature of the boundaries is regulated to $276 \mathrm{~K}$ and as a result pressure in 
the system equals 3.55 MPa (hydrate equilibrium). The initial pressure and temperature are uniform in the sediment.

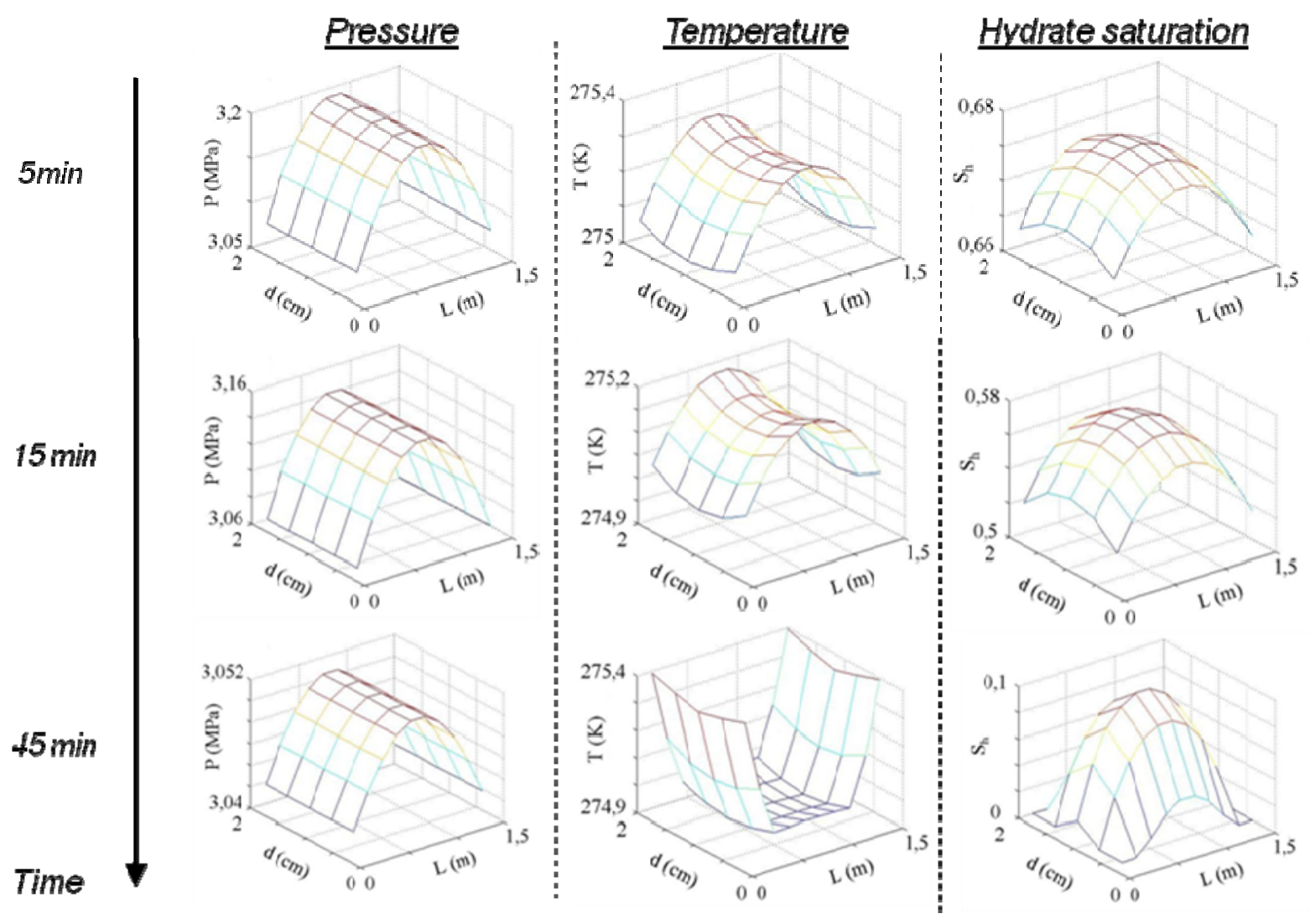

Fig 9: Evolution of pressure, temperature and hydrate saturation with time $\left(S_{h}{ }^{0}=0.7, S_{g}{ }^{0}=0.3, P_{d}=\right.$ $3.04 \mathrm{MPa}, K_{0}=10^{-13} \mathrm{~m}^{2}, \lambda_{s}=1.4 \mathrm{~W} \cdot \mathrm{m}^{-1} \cdot \mathrm{K}^{-1}$ )

Simulation results are shown as 2D plots (length vs diameter) representing a radial surface of the sediment core, hence a rectangular section of the system. The two axes have been rescaled for clarity. Figures present two symmetries: a longitudinal one due to the symmetry of the process (here a depressurization) and a radial one due to the uniformity of boundaries conditions. The parameter $\Delta P m$ used in the following is defined as the difference between the equilibrium pressure $P_{e}$ (before dissociation) and the pressure of dissociation $P_{d}$.

Fig. 8 and Fig. 9 record the heterogeneous changes in pressure, temperature and hydrate saturation as a function of time. For an absolute permeability $K_{0}$ of $10^{-11} \mathrm{~m}^{2}$ the hydrate saturation and temperature gradients display a radial symmetry; they record a "shrinkingcore" dissociation driven by the radial heat transfers between the external (hot) boundaries (Fig. 8). Indeed, when the depressurization takes place, methane hydrates become unstable and tend to reach a new local equilibrium by dissociating and a temperature decrease is observed. As the wall is maintained at a constant temperature, a radial temperature grandient appears and generates a heat flux: methane hydrates closed to the wall dissociate earlier than those present in the center of the core. Pressure remains almost uniform during the process and close to the pressure of dissociation $P_{d}$ that is imposed at the end of the tube.

For a lower permeability of $\mathrm{K}_{0}=10^{-13} \mathrm{~m}^{2}$, a contrasting behavior is simulated. The pressure becomes not uniform and a longitudinal gradient appears during the dissociation (Fig. 9). Longitudinal temperature gradients are stronger than radial gradients: overall, temperature, pressure and hydrate saturation depend mainly of time and longitudinal position. Methane 
hydrates closest to the two extremities dissociate first and the hydrate zone boundary switches from a "shrinking-core" behavior to a merely "longitudinal regression". The evolution of the temperature follows the evolution of the pressure: the decline is very slow and moderate during the dissociation (Fig. 9). The low permeability $\mathrm{K}_{\mathrm{o}}$ of the sediment limits the two phases flow (gas plus liquid water) resulting from the dissociation of methane hydrates. The dissociation is limited by the convection.

The influence of the absolute permeability $K_{0}$ on the kinetics of dissociation is even clearer when considering the hydrate half-life $t_{1 / 2}$, i.e. the time required to dissociate half of the hydrates. The dependence of $t_{1 / 2}$ on the absolute permeability $K_{0}$ and the ratio of $L / d$ can be calculated. For $K_{\mathrm{o}}$ in the range $10^{-13}-5 \times 10^{-11} \mathrm{~m}^{2}, t_{1 / 2}$ varies with the length and the diameter of the sediment core.

For $L / d=12$, the absolute permeability has no more influence on the kinetics of dissociation: $t_{1 / 2}$ is about $23 \mathrm{~min}$ whatever $K_{0}$. If the $L / d$ ratio increases to 25, a little slowdown appears (24 min) for an absolute permeability of $5 \times 10^{-13} \mathrm{~m}^{2}$. This slowdown was further confirmed by the evolution of $t_{1 / 2}$ for a higher ratio. For a $L / d$ ratio of 50 , a transition is found for an absolute permeability $K_{\mathrm{o}}$ below $10^{-12} \mathrm{~m}^{2}$ : between $10^{-12}$ and $10^{-13} \mathrm{~m}^{2}$ the time of half dissociation $t_{1 / 2}$ increases from 23 to $26 \mathrm{~min}$. Overall, this figure highlights the transition between two dissociation modes for a sediment core partly saturated with methane hydrate: one where the absolute permeability $K_{\mathrm{o}}$ does not influence the kinetics of dissociation and another one where the decrease of $K_{\mathrm{o}}$ increases the time of dissociation.

\section{Intermediate conclusion}

A 2D model for methane hydrate decomposition in a sediment core was presented above. It was tested at core scale with physical values available in the literature. The simulator allowed us to track the evolution of pressure, temperature and hydrate saturation in the sediment during the dissociation, and two different dissociation modes appeared, one governed by heat transfers, another governed by mass transfers. The transition between these modes is sensitive to the absolute permeability $\mathrm{K}_{\mathrm{o}}$ and the initial hydrate saturation $\mathrm{S}_{\mathrm{h}}^{0}$ i.e. two parameters that influence the overall dissociation kinetics. So, for a ratio $L / d$ of 100 , this transition takes place for permeability $K_{\mathrm{o}}$ around $5 \times 10^{-12} \mathrm{~m}^{2}$ and a saturation $\mathrm{S}_{\mathrm{h}}^{0}$ around 0.5 (Fig. 10).

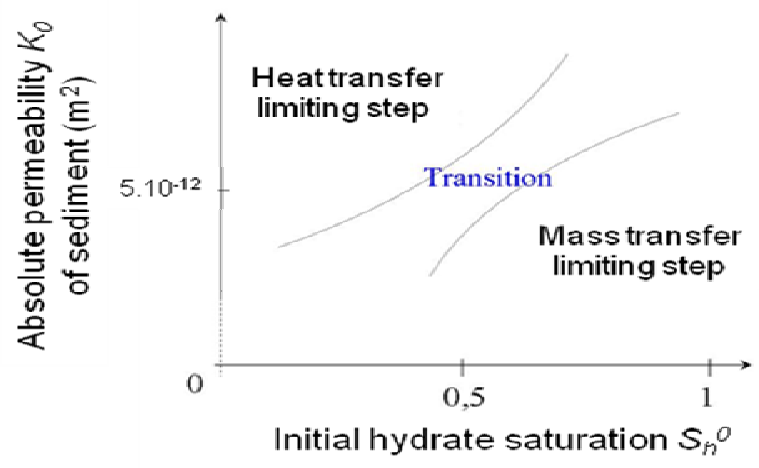

Fig 10: Limiting steps of methane hydrate dissociation inside $(L / d=100)$.

The next stage of the study is the validation of the model through experiments and the comparison of predicted with experimental results. 


\section{Experimental approach}

\section{Experimental set-up}

In the previous part, a transition has been observed between a heat and a mass transfer limiting step during the methane hydrate dissociation inside a sediment core. Since the length of the core is a key parameter in this transition, the experimental set-up consists of five cylindrical segments having the same diameter (1/2 inch) but different lengths, for a total (Fig. 11) length of $2.6 \mathrm{~m}$ (Fig. 12). Each zone is temperature and pressure controlled, equipped with pressure transducers and can be isolated from the others. At the right extremity of the line, a methane bottle allows to rise the gas pressure in the system; at the left extremity, a calibrated expansion volume is installed (a ballast and a solenoid valve which controlled the pressure during the dissociation). A special set-up was developed for the temperature probes (pt10o) to penetrate in the sediment zones, and to measure temperature while allowing liquid and gas transfers (Fig. 11).

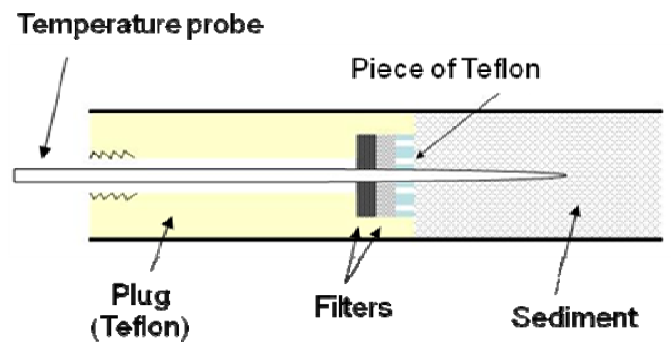

Fig 11: Sediment zone boundaries.
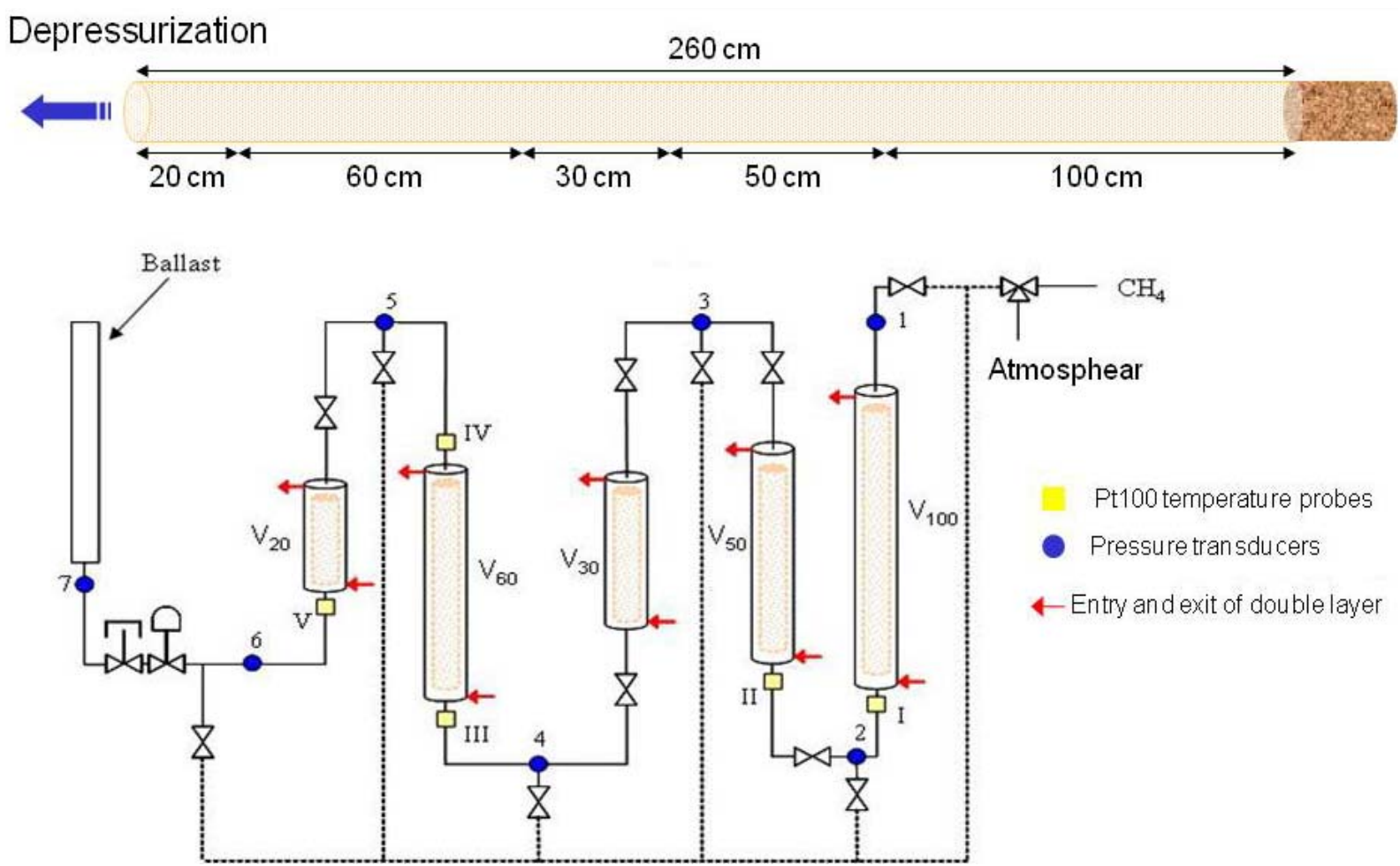

Fig 12: Experimental set-up.

Each side of sediment zone is equipped with a end piece composed of a filter and a Teflon element (Fig. 11). Filters (with a mesh size between 200 and $10 \mu \mathrm{m}$ ) prevent the sediment to leak outside the tubes. Teflon material is firstly used in this experiment in order to limit the longitudinal heat transfer between the sediment zones and the "dead" zones (i.e. connections 
between sediment zones). Secondly, by screwing the last element of the tube to the tube (the piece does not appear on (Fig. 11), the Teflon element is pushed against the filter piece and compact the sediment. Also, the Teflon piece and filter allows to enter a temperature probe.

Procedure and sediment types

The flexibility of the set-up allows an easy change of sediment between two experiments. Five different sediments have been used in order to vary the absolute permeability $K_{0}$ and the heat conductivity $\lambda_{s}$ of the medium in which methane hydrate is crystallized and dissociated. Table 1 gives the permeability values of the different sediments and their average grain diameter as measured with a MALVERN granulometer. These sediments were chosen to display absolute permeabilities $K_{0}$ between $10^{-10}$ and $10^{-13} \mathrm{~m}^{2}$. The three different types of micro-balls have the same composition, only the average grain diameter is different: these balls are mainly composed ( $75 \%$ molar) of $\mathrm{SiO}_{2}$. They have been bought to the MINERALEX society. Natural sand was provided by Pr. Chuvilin (University of Moscow); it was collected from the region of Moscow (upper Jurassic) and is mainly composed of quartz.

Table 1: Characterization of sediments.

\begin{tabular}{|c|c|c|c|c|c|}
\hline Sediment & Sand & Silica & $\begin{array}{c}\text { Micro-balls } \\
\text { type } 1\end{array}$ & $\begin{array}{c}\text { Micro-balls } \\
\text { type } 2\end{array}$ & $\begin{array}{c}\text { Micro-balls } \\
\text { type } 3\end{array}$ \\
\hline $\begin{array}{c}\text { Absolute } \\
\text { permeability } K_{0}\left(\mathrm{~m}^{2}\right) \\
\text { "Bear correlation" }\end{array}$ & $4 \times 10^{-11}$ & $3 \times 10^{-11}$ & $2 \times 10^{-10}$ & $5 \times 10^{-12}$ & $5^{\times 10^{-13}}$ \\
\hline $\begin{array}{l}\text { Heat conductivity } \\
\left(\mathrm{W} \mathrm{m}^{-1} \mathrm{~K}^{-1}\right)\end{array}$ & 11 & $1-1.4$ & $1-1.4$ & $1-1.4$ & $1-1.4$ \\
\hline $\begin{array}{l}\text { Average grain } \\
\text { diameter }(\mu \mathrm{m})\end{array}$ & 255 & 205 & 645 & 90 & 30 \\
\hline
\end{tabular}

Each experiment starts with crystallizing a hydrate phase in a wet porous medium. Wetting of sediment is performed by mixing sand and ice crystals, then ice is melted and the wetted sand is introduced in the tubes. Then, water is converted into hydrates as the methane pressure is increases to $10 \mathrm{MPa}$ and the temperature maintained at $276 \mathrm{~K}$. A delay of $48 \mathrm{~h}$ is needed for the apparent end of hydrate formation: after $48 \mathrm{~h}$, gas consumption becomes null (i.e. pressure decrease stops. But, a mass balance calculation allows us to evaluate the water conversion into hydrate to be $70 \%$. Then, pressure is decreased to the hydrate equilibrium pressure corresponding to the operative temperature.

From this point, the dissociation can be started. The pressure at one end of the pipe(s) section is reduced to a operative value $P_{d}$ : the difference between the equilibrium pressure $P_{e q}$ and the operative pressure $P_{d}$ (also called dissociation pressure) is the driving force $\Delta P m$. The other side of the pipe(s) section is closed. A solenoid valve (left end of the set-up, Fig. 12) allows the pressure to be controlled at the value $P_{d}$ by opening and closing the pipe and evacuating the gas to ballast. So the kinetics of dissociation is monitored by measuring the pressure rise in the ballast. Fig. 13 gives an overview of the experimental set-up, with the five sediment zones, their cooling jackets, and the methane supply line. The computer on the right side allows the acquisition of $\mathrm{P} \mathrm{T}$ data and the regulation of the solenoid valve during the dissociation of methane hydrate.

Results

One key parameter of the hydrate dissociation is the driving force $\Delta P m$ applied to the sediment core. Next figures illustrate the bulk degree of conversion $\chi$ for the dissociation, as defined by 


$$
\chi=P_{\text {ballast }}(t) / P_{\text {ballast }}(t \rightarrow \infty)
$$

so this value varies between $\mathrm{o}$ and 1 (at the end of the dissociation).

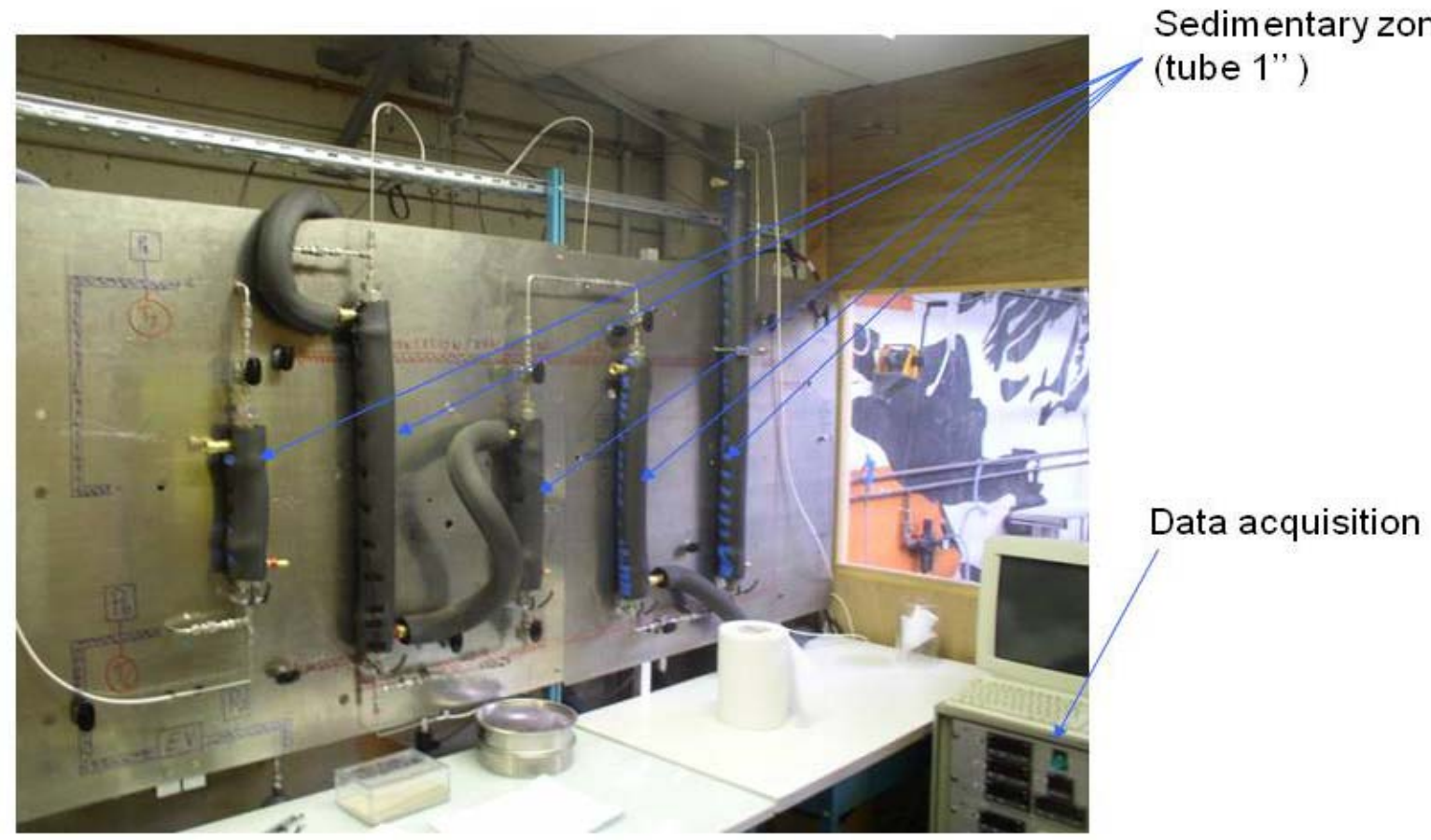

Fig 13: Picture of the laboratory.

The kinetics of dissociation is obviously not linear with respect to time: dissociation rate is quickest at the beginning and then decreases (Fig. 14) for large timescales. The driving force $\Delta P m$ influences directly the kinetics of dissociation: an increase of $\Delta P m$ enhances the kinetics.
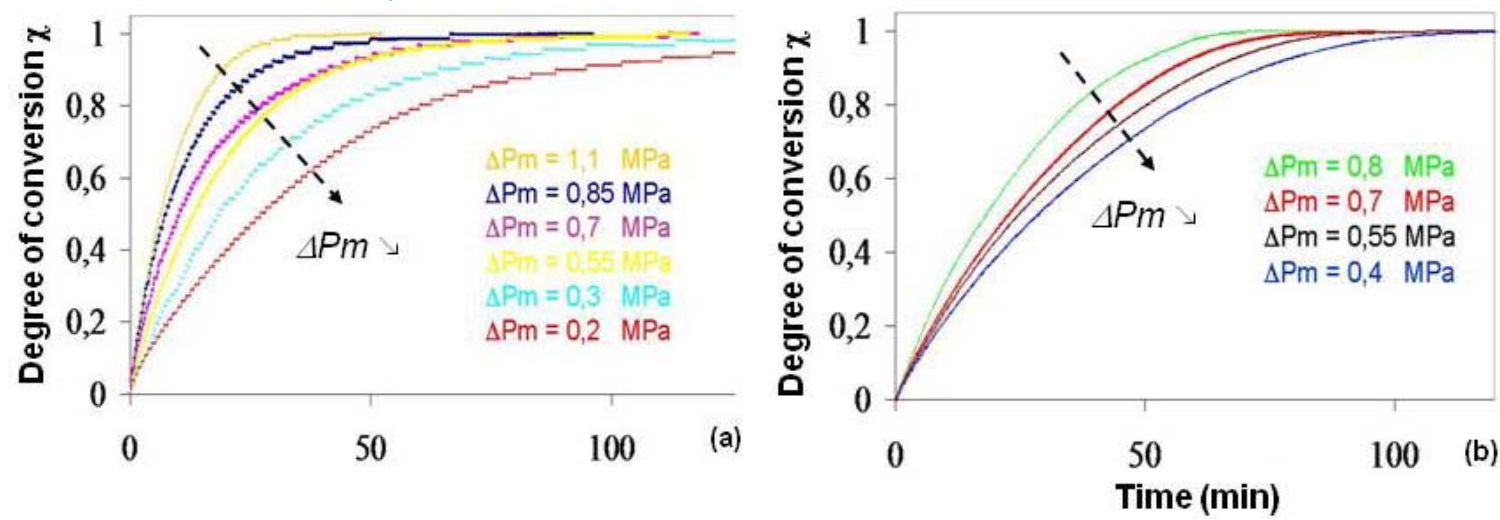

Fig 14: Impact of driving force $\triangle P m$ on the degree of conversion $\chi:$ (a) silica and (b) micro-balls type 3.

For instance, for silica sand, a decrease of the driving force strongly lowers the dissociation kinetics (Fig. 14(a)): for same initial conditions, when $\Delta \mathrm{Pm}$ evolves from 1.1 to $0.2 \mathrm{MPa}$, the time of dissociation increases from 30 to $140 \mathrm{~min}$.

The same observation applies for micro-balls type 3, but the impact is less important: a decrease in $\Delta P m$ induces again a slowdown but in a moderate way in comparison to silica sand.

Moreover, the numerical simulations predict that heat conductivity $\lambda_{s}$ of the sediment must also influence the kinetics. For two sediments (natural sand and silica sand) which have the same absolute permeability $K_{\mathrm{o}}$ but different heat conductivities $\lambda_{s}$ the degree of conversion $\chi$ 
should vary differently. Indeed, for a driving force of 0.7 , the dissociation kinetics is faster with natural than with silica sand (Fig. 15); the same result is obtained for a smaller driving force: an increase of the heat conductivity accelerates the dissociation but this increase is not linear. This conclusion is mostly valuable for the case of sediment with a high permeability $\left(K_{0}>5 \times 10^{-12} \mathrm{~m}^{2}\right)$ where the hydrate dissociation limiting step is heat transfer.

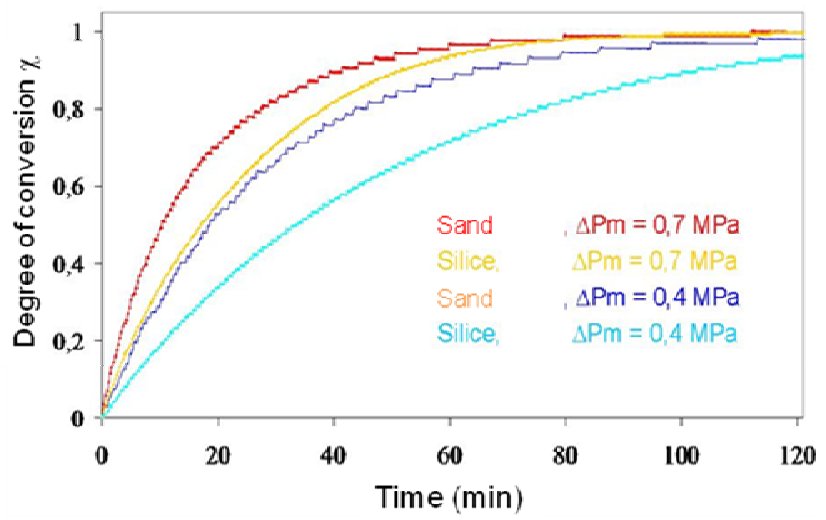

Fig 15: Impact of heat conductivity $\lambda_{s}$ on dissociation.

All the experimental results have been obtained by crystallizing first the hydrate from liquid water. The value $S_{\mathrm{w}}^{\text {init }}$ in our experiments is thus determined by the quantity of water introduced in the sediment prior to hydrate formation.

An important issue in the experiments is whether or not temperature or pressure gradients are generated during the dissociation inside the sediment core.

For high permeability sediments $\left(K_{0}>5 \times 10^{-12} \mathrm{~m}^{2}\right.$, Fig. 16, left side) and whatever the initial water saturation $S_{\mathrm{w}}^{\text {init }}$ we do not record pressure gradient in the system: when the depressurization is applied to one side (P6), the same pressure is measured at the other side of the core (P1). Similarly, the different temperature probes (in the center of the tubes) evolve at the same rate. The dissociation rate is controlled by heat transfer from the surrounding, i.e. from the cooling jackets (Fig. 16, left side).

For low permeability sediment $\left(K_{0}<5 \times 10^{-12} \mathrm{~m}^{2}\right.$, Fig. 16 , right side $)$ a very different behavior is observed. Both pressure and longitudinal temperature gradients can be observed. At the beginning of the dissociation (when the pressure P6 decreases), the pressure P1 and P2 do not follow the same trend, and a longitudinal pressure gradient is observed and a two phase flow is generated in response to this gradient. In fact, during this early stage of the dissociation, the sediment is almost impermeable and the depressurization cannot be transmitted to the whole core. The hydrate dissociates first at the extremity where the depressurization has been applied, and the dissociation migrates towards the other side: dissociation is governed by longitudinal effects and mass transfer (Fig. 16, right side). As a consequence, temperature adjusts to equilibrium temperature (that is lower than the boundary condition) and a longitudinal temperature gradient appears. Indeed, temperature probes close to the extremity of depressurization ( $\mathrm{T}_{5}$ and $\mathrm{T}_{3}$, cf. Fig. 12) come back to the operative condition first $(\mathrm{T}=276 \mathrm{~K})$ after full dissociation. At the opposite extremity, dissociation ends later, so the temperature probe T1 (cf. Fig. 12) comes back to the operative temperature later.

The initial water saturation $\mathrm{S}_{\mathrm{w}}^{\text {init }}$ has also a great impact on the gradients. For a high $\mathrm{S}_{\mathrm{w}}^{\text {init }}$ the local permeability $K$ can be decreased significantly, and this induces important pressure gradients. 


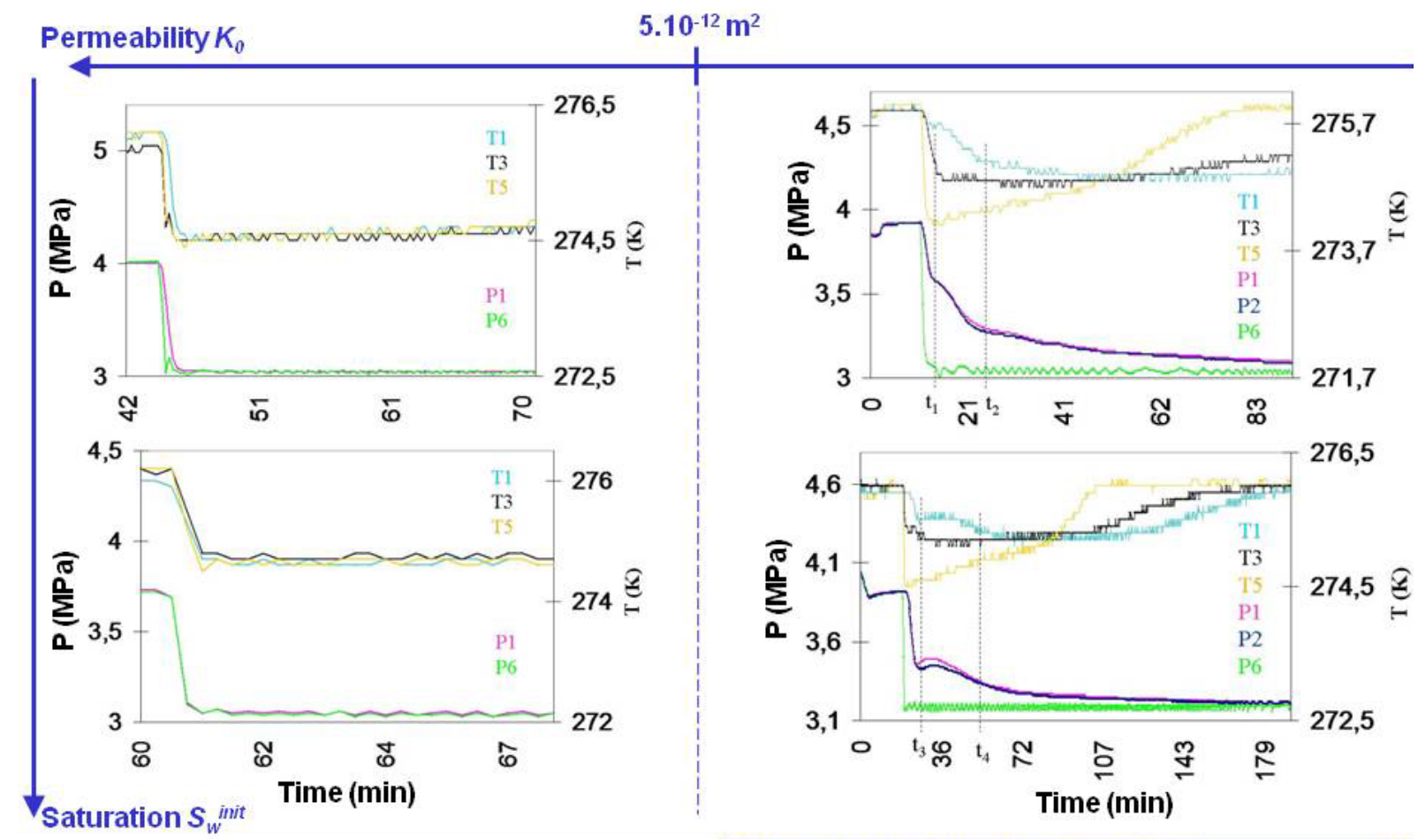

Fig 16: Influence of absolute permeability $K_{o}$ and initial water saturation $\mathrm{S}_{\mathrm{w}}^{\text {init }}$ on pressure and temperature inside the cores during the dissociation (for the location of P1, P6, T1, etc. see Fig. 12).

Sand, silica, micro-balls type 1
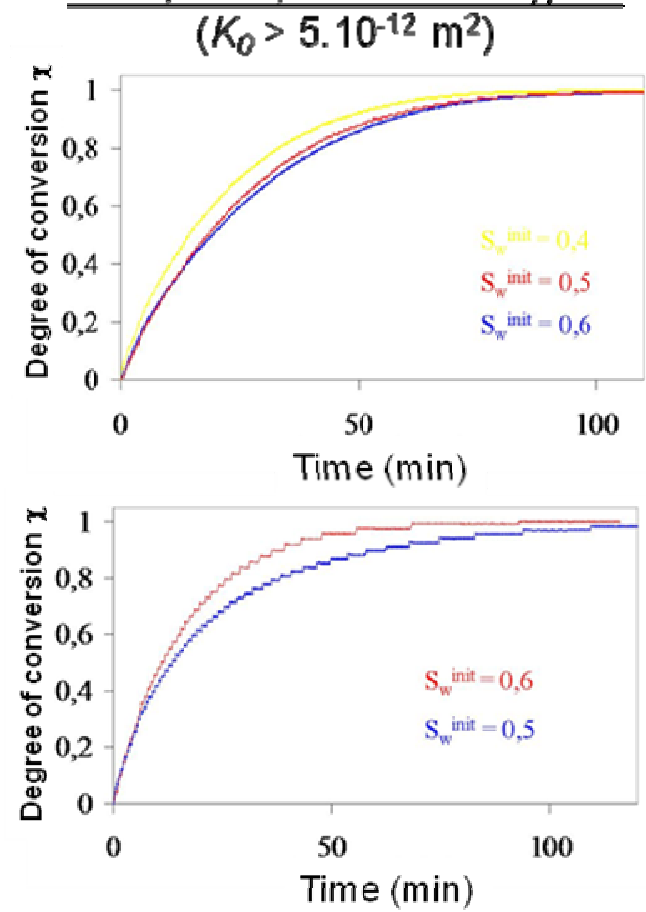

Micro-balls type 2 and 3 $\left(K_{0}<5.10^{-12} \mathrm{~m}^{2}\right)$
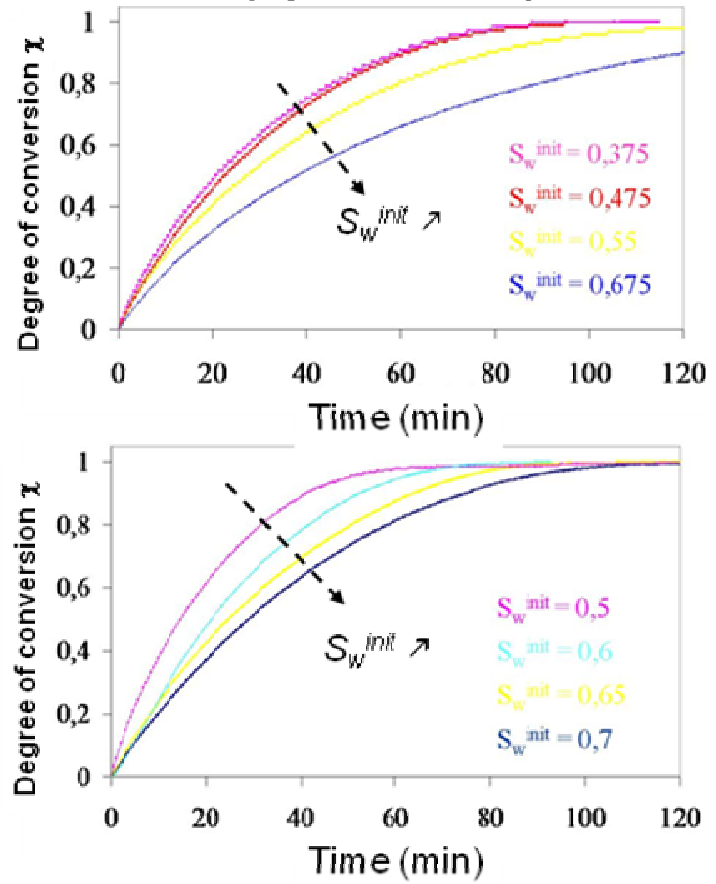

Fig 17: Influence of initial water saturation $\mathrm{S}_{\mathrm{w}}^{\mathrm{init}}$ on the degree of conversion $\chi$.

Two different regimes appear by changing initial water (or hydrate) saturation: 
* For high permeability sediments (sand, silica or micro-balls type 1), the initial water saturation $S_{\mathrm{w}}^{\mathrm{init}}$ has little influence on the dissociation kinetics (Fig. 17, left side). An increase or decrease of $\mathrm{S}_{\mathrm{w}}^{\text {init }}$ does not significantly change the evolution of $\chi$ with time: the dissociation rate is limited by heat transfer.

* Oppositely, for low permeability sediments $\left(K_{0}<5 \times 10^{-12} \mathrm{~m}^{2}\right)$, the initial water saturation $\mathrm{S}_{\mathrm{w}}^{\text {init }}$ is a key parameter (Fig. 17, right side). By increasing the initial water saturation $\mathrm{S}_{\mathrm{w}}^{\mathrm{init}}$ (and thus initial hydrate saturation), the dissociation rate is decreased. For example, with micro-balls of type 3, varying $S_{w}^{\text {init }}$ from 0.5 to 0.7 , increases the dissociation time from 50 to $100 \mathrm{~min}$ (Fig. 17, bottom left).

This result can be explained by a mass transfer limitation in low permeability sediments: the influence of $\mathrm{S}_{\mathrm{w}}^{\text {init }}$ is most pronounced for the sediment with lowest permeability (micro-balls type 3).

The half dissociation time is another parameter that allows comparing the model predictions and the experimental results. The dependence of $t_{1 / 2}$ versus the driving force $\Delta P m$ confirms the previous results (Fig. 18), as it decreases with increasing the driving force:

* For high permeability sediments (sand and silica), $t_{1 / 2}$ depends on the heat conductivity $\lambda_{s}$ of the sediment: $t_{1 / 2}$ decreases with increasing $\lambda_{s}$. But $t_{1 / 2}$ varies little with permeability $K_{0}$ and the dissociation is only controlled by the heat transfers.

* For low permeability sediments, the absolute permeability $K_{\mathrm{o}}$ (and more generally mass transfer) tunes the evolution of $t_{1 / 2}$ as a function of the driving force: an increase of $K_{0}$ slowdowns the dissociation rate. For a same initial water saturation, an evolution of $K_{\mathrm{o}}$ from $3 \times 10^{-11}$ to $5 \times 10^{-13} \mathrm{~m}^{2}$ results in an increase of $t_{1 / 2}$ from 25 to 45 min (Fig. 18).

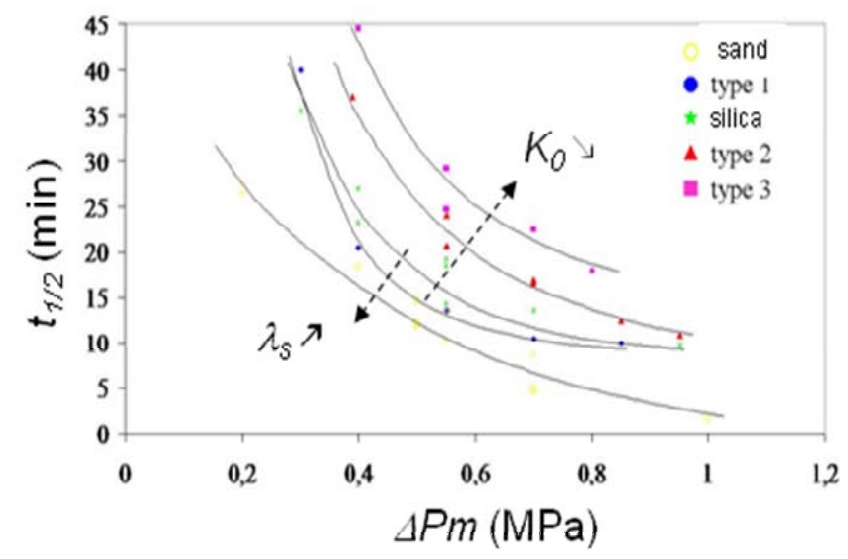

Fig 18: Evolution of $t_{1 / 2}$ with driving force $\Delta P m\left(\mathrm{~S}_{\mathrm{w}}^{\mathrm{init}}=0.7\right)$.

\section{Agreement with simulations}

We turn now to the quantitative agreement between model predictions and experimental results.

The first issue is whether or not the experimental gradients agree with the calculated ones. Fig. 19 plots the evolution of $\mathrm{P}_{\mathrm{e}}$ (the pressure at the extremity of the core $(=\mathrm{P} 6)$ ) and $\mathrm{P}_{\mathrm{c}}$ (the 
pressure at the other extremity $(=P 1))$. For these two pressures numerical and experimental results are comparable and follow the same trend.

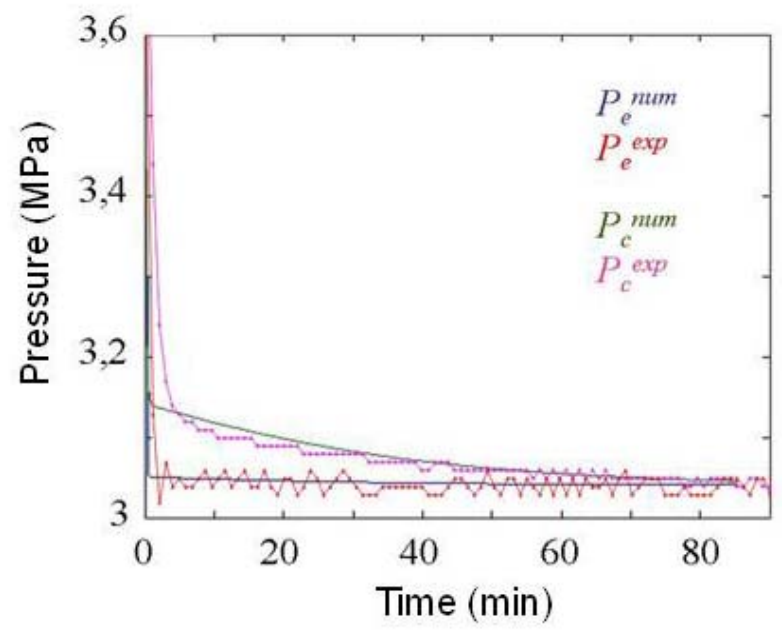

Fig 19: Evolution of different pressures with time (-numerical, …e experimental) $S_{h}{ }^{o}=0,5, S_{g}{ }^{o}=0,3$, $P_{d}=3,05 \mathrm{MPa}, K_{o}=5.10^{-12} \mathrm{~m}^{2}, \lambda_{s}=1,4 \mathrm{~W} \cdot \mathrm{m}^{-1} \cdot \mathrm{K}^{-1}, \beta=3$

The next point is the quality of the fit between calculated and measured degrees of conversion $\chi$. For a similar system (i.e. same initial and boundary conditions) the same trend is observed for experimental and numerical results (Fig. 20).

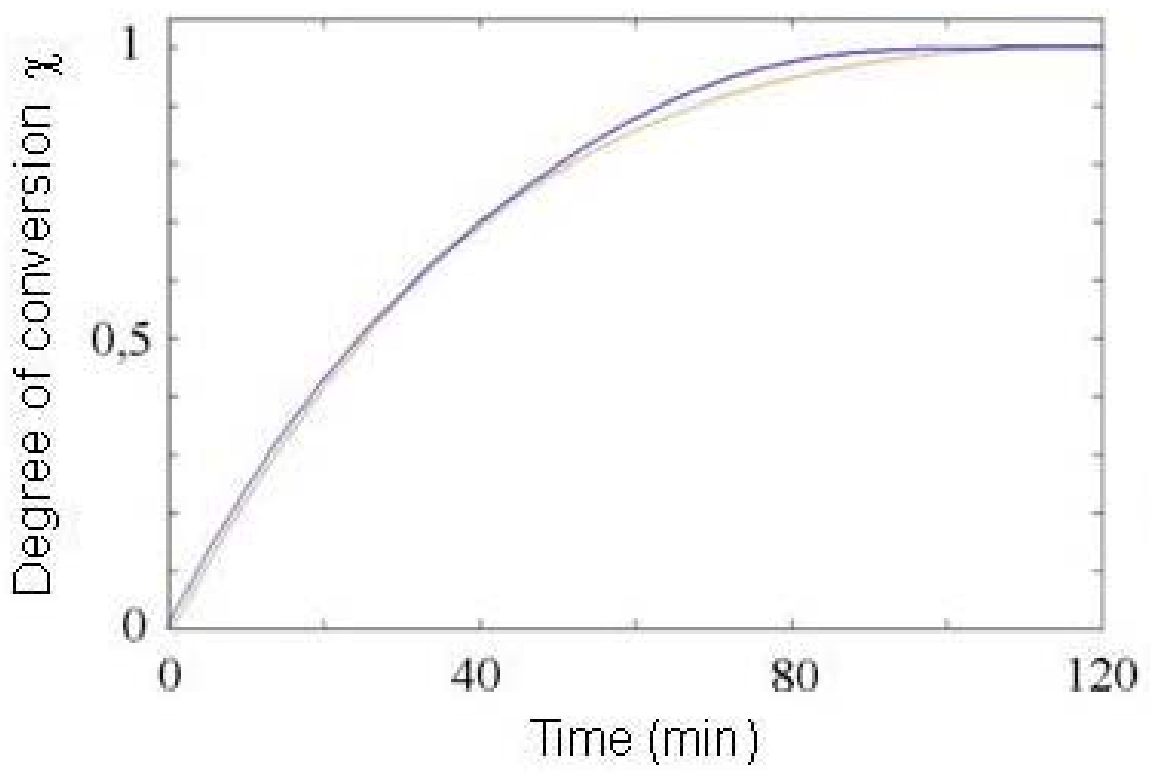

Fig 20: Comparison of the evolution of the degree of conversion with time (- experimental, numerical) $S_{h}{ }^{o}=0,42, S_{g}{ }^{o}=0,4, P_{d}=3,05 \mathrm{MPa}, K_{o}=5 \cdot 1 \mathrm{O}^{-13} \mathrm{~m}^{2}, \lambda_{s}=1,4 \mathrm{~W} \cdot \mathrm{m}^{-1} \cdot \mathrm{K}^{-1}, \beta=3$

Overall, the agreement between experimental and numerical results is excellent. Fig. 21 shows the comparison of the half-times of dissociation (t1/2) calculated and measured: all experimental values are in the range $\pm 10 \%$ and the standard deviation is less than $5 \%$.

This agreement confirms the overall validity of the numerical model. 


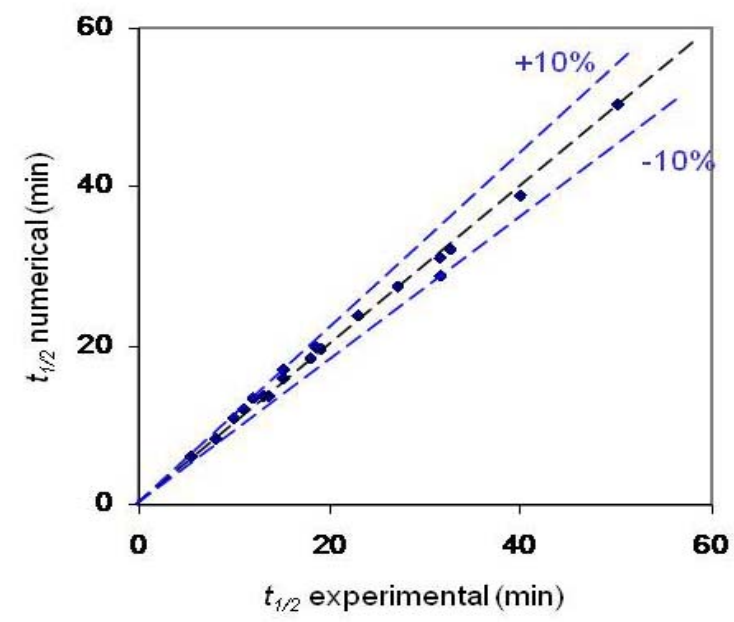

Fig 21: Comparison between numerical and experimental results $(\beta=3)$.

\section{Reproducibility}

The question of the sample integrity during formation and dissociation has not been studied in detail in this study. The main reason is that our experiment do not allow to monitor the microscopic level (i.e. the pore level), but only macroscopic state, via pressure and temperature monitoring. But, based on the results of the Fig. 21, we can deduce that our samples maintain their integrity between two experiments. In fact, each sample (four samples have been studied, Table 1) has been experimented in various conditions of pressure without changing the substrate between experiments. So, a same substrate has encountered many cycle of formation and dissociation. Based on the good agreement between the model and the experimental results (for example Fig. 21), we can conclude that the physical properties of the sample do not change between two experiments, i.e. the initial permeability and the initial heat conductivity remain constant from cycle to cycle. In first approximation, we can exclude the occurrence of deep modifications (such as short-cuts, or segregation of water) of the substrate.

\section{Limitations and conclusions}

It must be noticed first that the application of the above results to field productivity evaluation suffers some limitations:

* The experimental work was completed on loose sand packs rather than on cohesive sandstones, so the damaging effects of hydrate formation and dissociation cycles on the porous medium could not be evaluated.

* Experimental boundary conditions (jacketed cores) do not allow compactional effects due to methane production to be assessed.

* Low permeability sediments (fine sands, silts) are often clay-rich rather than similar to glass beads in terms of wettability and pore shape, so the models used here to represent relative permeabilities are crude approximations at best.

Given these limitations, the dissociation kinetics of methane hydrate inside sediment core appears to be controlled by different limiting steps depending on the initial characteristics of the sediment. The numerical approach proposed in the first part of this work evidences two contrasting modes of dissociation: a mass transfer limited mode for low permeability sediments, and a heat transfer limited mode for high permeability sediments. 
The numerical model allowed us to design an experimental device that is presented in the second part of the paper, together with a procedure for the controlled formation and dissociation of methane hydrate.

Experimental results document the influence of key parameters such as the driving force $\Delta P m$, heat conductivity $\lambda_{s}$, and permeability $K_{o}$ of the sediment.

A distinction is established between a dissociation governed by heat transfers and another one governed by mass transfers: for high permeability sediments, the heat conductivity $\lambda_{s}$ of the sediment is a key parameter that controls the dissociation kinetics. For low permeability sediment $\left(K_{0}<5 \times 10^{-12} \mathrm{~m}^{2}\right)$, the dissociation kinetics becomes dependent on the parameters that influence mass transfers, i.e. the initial hydrate saturation $\mathrm{S}_{\mathrm{h}}^{0}$ or the absolute permeability $K_{\mathrm{o}}$ of the sediment.

It needs to be noted here that the limitation is never the intrinsic rate of dissociation at the crystal level.

The numerical model presented in the first part, and the experimental results presented in the second part are in very good agreement, both qualitatively and quantitatively.

\section{Notation}

$\mathrm{D}_{\mathrm{m}, \mathrm{e}} \quad$ diffusion coefficient of methane (water)

$\mathrm{E}^{\mathrm{j}} \quad$ internal energy

g gravity acceleration

$\mathrm{h}_{\mathrm{j}} \quad$ specific enthalpy of component $\mathrm{j}$

$\mathrm{j} \quad$ specific internal energy of component $\mathrm{j}$

$\mathrm{k}_{\mathrm{rl}} \quad$ relative permeability of liquid

$\mathrm{k}_{\mathrm{rg}} \quad$ relative permeability of gas

$\mathrm{K}, \mathrm{K}_{\mathrm{o}} \quad$ absolute permeability

$\mathrm{M}^{\mathrm{j}} \quad$ molecular weight of component $\mathrm{j}$

$p \quad$ pressure

$\Delta \mathrm{Pm} \quad$ driving force

Q thermal energy flux

$R \quad$ ideal gas constant

$\mathrm{S}_{\mathrm{h}} \quad$ hydrate saturation

$\mathrm{S}_{\mathrm{l}}\left(\mathrm{S}_{\mathrm{w}}\right) \quad$ liquid saturation

$\mathrm{S}_{\mathrm{g}} \quad$ gas saturation

$\mathrm{t}_{1 / 2} \quad$ time of half dissociation

$T \quad$ temperature

$\mathrm{v}_{\mathrm{j}} \quad$ volume fraction of the component $\mathrm{j}$

$\mathrm{W}_{\mathrm{V}} \quad$ work related to volume change

$\mathrm{W}_{\mathrm{D}}$ dissipation work

$\mathrm{xl}_{\mathrm{j}}^{\mathrm{j}} \quad$ mass fraction of component $\mathrm{j}$ in the liquid phase

\section{Greek letters}

$\beta \quad$ Civan parameter

$\gamma_{1}, \gamma_{\mathrm{g}}, \gamma_{\mathrm{h}}$, net mass production rate of water, methane and hydrate due to dissociation

$\lambda \quad$ heat conductivity

$\mu_{1}, \mu_{\mathrm{g}} \quad$ liquid and gas viscosity

$\rho_{\mathrm{l}}, \rho_{\mathrm{g}}, \rho_{\mathrm{h}} \quad$ liquid, gas and hydrate mass density

$\Phi_{\mathrm{o}} \quad$ porosity 
$\begin{array}{ll}\Phi_{\mathrm{e}} & \text { effective porosity } \\ \chi & \text { degree of conversion }\end{array}$

$\begin{array}{ll}\text { Sub/Superscripts } \\ \text { e } & \text { water } \\ \text { l } & \text { liquid } \\ \text { h } & \text { hydrate } \\ \text { g } & \text { gaz } \\ \text { m } & \text { methane } \\ \text { s } & \text { sediment }\end{array}$

\section{Acknowledgments}

We are grateful to Daniel Garcia for his useful suggestions and corrections. This work was supported by the INTAS European project coordinated by the professor Kuhs of the Göttingen University.

\section{References}

Anderson, R. ; Burgass, R. W. ; Tohidi, B. ; Østergaard, K K. (11-15 Juin 2001) A new experimental set-up for the study of formation and dissociation of methane hydrates in sediments, 63rd EAGE conference and Exhibition, P567, Amsterdam, PaysBas

Ahmadi, G.; Ji, C.; Smith, D. H. Numerical solution for natural gas production from methane hydrate dissociation. $J$ Pet Sci Eng 2004, 41 (4), 269-285.

Bastian, P. Numerical computation of multiphase flows in porous media. Technische Fakultät der Christian-Albrechts-Universität Kiel, Habilitation thesis, 1999.

Bonnefoy, O. Influence de cristaux d'hydrates de gaz ou de glace sur la perméabilité d'un milieu poreux, Ecole des Mines de St-Etienne, Habilitation thesis, 2005.

Chen, Z. X.; Huan, G. R.; Li, B. Y. An improved IMPES method for two-phase flow in porous media. Transp. Porous Media 2004, 54 (3), 361-376.

Chounet, L. M.; Hilhorst, D.; Jouron, C.; Kelanemer, Y.; Nicolas, P. Simulation of water flow and heat transfer in soils by means of a mixed finite element method. Adv. Water Resour. 1999, 22 (5), 445-46o.

Class, H. Theorie und numerische modellierung nichtisothermer mehrphasenprozesse in NAPL-kontaminierten porösen medien, Institut Wasserbau, Universität Stuttgart, Habilitation thesis, 2001

Class, H.; Helmig, R.; Bastian, P. Numerical simulation of non-isothermal multiphase multicomponent processes in porous media. 1. An efficient solution technique. Adv. Water Resour. 2002, 25 (5), 533-550.

Dawson, C. N.; Klie, H.; Wheeler, M. F.; Woodward, C. S. A parallel , implicit , cell-centered method for two-phase flow with a preconditioned Newton-Krylov solver. Journal on Computational Geosciences 1997, 1, 215-249.

Durgut, I.; Parlaktuna, M. A numerical method for the gas production process in gas hydrate reservoirs. $2^{\text {nd }}$ International Conference on Natural Gas Hydrates, Toulouse, France, June 2-6, 1996.

Henninges, J .; Schrötter, J •; Erbas, K.; Huenges, E. Temperature field of the Mallik gas hydrate occurrence. Implications on phase changes and thermal properties, GEO Technologien 2002. 
Herri, J . Etude de la formation de l'hydrate de méthane par turbidimétrie in situ, Ecole des Mines de St-Etienne, Habilitation thesis, 1996.

Holstad, A. Temperature-driven fluid flow in porous media using a mixed finite element method and a finite volume method. Adv. Water Resour. 2001, 24 (8), 843862.

Ji, C.; Ahmadi, G.; Smith, D. H. Natural gas production from hydrate decomposition by depressurization. Chem. Eng. Sci. 2001, 56 (20), 5801-5814.

Kim, H. C.; Bishnoi, P. R.; Heidemann, R. A.; Rizvi, S. S. H. Kinetics of methane hydrate decomposition. Chemical engineering science 1987, 42 (7), 16451653 .

Kneafsey, T. J. ; Tomutsa, L. ; Moridis, G. J . ; Seol, Y. ; Freifeld, B. M. ; Taylor, C. E. ; Gupta, A. Methane hydrate formation and dissociation in a partially saturated core-scale sand sample Journal of Petroleum Science \& Engineering 2007, 56, 108-126.

Larsen, R.; Habetinova, E.; Holm, H. A simple hydrate plug dissociation model with permeability effects. Fifth International Conference on Gas Hydrates, Trondheïm, Norway, June 13-16, 2005.

Moridis, G. J.; Sloan, E. D. Gas production potential low-saturation hydrate accumulations in oceanic sediments Energy Conversion \& Management 2007, 48, 1834-1849.

Nguyen Hong, D. Dissociation des bouchons d'hydrates de gaz dans les conduites pétrolières sous-marines, Ecole des Mines de St-Etienne, Habilitation thesis, 2005.

Rutqvist, J . ; Moridis, G.J • ; Grover, T. ; Collett, T. Geomechanical response of permafrost-associated hydrate deposits to depressurization-induced gas production. Journal of Petroleum Science \& Engineering 2009, in press.

Saad, Y. Iterative methods for sparse linear systems, SIAM Edition, 2000.

Sung, W. M.; Lee, H.; Lee, H.; Lee, C. Numerical study for production performances of a methane hydrate reservoir stimulated by inhibitor injection. Energy Sources 2002, 24 (6), 499-512.

Yousif, M. H.; Abass, H. H.; Selim, M. S.; Sloan, E. D. Experimental and theoretical investigation of methane gas hydrate dissociation in porous media, SPE Reservoir Engineering, 1991 